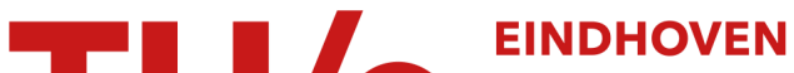 \\ UNIVERSITY OF \\ TECHNOLOGY
}

\section{Multiscale model of gradient evolution in turbulent flows}

Citation for published version (APA):

Biferale, L., Chevillard, L., Meneveau, C., \& Toschi, F. (2007). Multiscale model of gradient evolution in turbulent flows. Physical Review Letters, 98(21), 214501-1/4. [214501]. https://doi.org/10.1103/PhysRevLett.98.214501

DOI:

10.1103/PhysRevLett.98.214501

Document status and date:

Published: 01/01/2007

\section{Document Version:}

Publisher's PDF, also known as Version of Record (includes final page, issue and volume numbers)

\section{Please check the document version of this publication:}

- A submitted manuscript is the version of the article upon submission and before peer-review. There can be important differences between the submitted version and the official published version of record. People interested in the research are advised to contact the author for the final version of the publication, or visit the $\mathrm{DOI}$ to the publisher's website.

- The final author version and the galley proof are versions of the publication after peer review.

- The final published version features the final layout of the paper including the volume, issue and page numbers.

Link to publication

\section{General rights}

Copyright and moral rights for the publications made accessible in the public portal are retained by the authors and/or other copyright owners and it is a condition of accessing publications that users recognise and abide by the legal requirements associated with these rights.

- Users may download and print one copy of any publication from the public portal for the purpose of private study or research.

- You may not further distribute the material or use it for any profit-making activity or commercial gain

- You may freely distribute the URL identifying the publication in the public portal.

If the publication is distributed under the terms of Article 25fa of the Dutch Copyright Act, indicated by the "Taverne" license above, please follow below link for the End User Agreement:

www.tue.nl/taverne

Take down policy

If you believe that this document breaches copyright please contact us at:

openaccess@tue.nl

providing details and we will investigate your claim. 


\title{
Multiscale Model of Gradient Evolution in Turbulent Flows
}

\author{
Luca Biferale, ${ }^{1}$ Laurent Chevillard, ${ }^{2}$ Charles Meneveau, ${ }^{2}$ and Federico Toschi ${ }^{3}$ \\ ${ }^{1}$ Dipartimento Fisica and INFN, Università di “Tor Vergata," Via della Ricerca Scientifica 1, 00133 Roma, Italy \\ ${ }^{2}$ Department of Mechanical Engineering and Center for Environmental and Applied Fluid Mechanics, The Johns Hopkins University, \\ 3400 N. Charles Street, Baltimore, Maryland 21218, USA \\ ${ }^{3}$ Istituto per le Applicazioni del Calcolo CNR, Viale del Policlinico 137, 00161 Roma, Italy, \\ and INFN, Sezione di Ferrara, Via G. Saragat 1, I-44100 Ferrara, Italy
}

(Received 5 December 2006; published 21 May 2007)

\begin{abstract}
A multiscale model for the evolution of the velocity gradient tensor in turbulence is proposed. The model couples "restricted Euler" (RE) dynamics describing gradient self-stretching with a cascade model allowing energy exchange between scales. We show that inclusion of the cascade process is sufficient to regularize the finite-time singularity of the RE dynamics. Also, the model retains geometrical features of real turbulence such as preferential alignments of vorticity and joint statistics of gradient tensor invariants. Furthermore, gradient fluctuations are non-Gaussian, skewed in the longitudinal case, and derivative flatness coefficients are in good agreement with experimental data.
\end{abstract}

DOI: 10.1103/PhysRevLett.98.214501

PACS numbers: 47.27.- $\mathrm{i}, 47.10 .-\mathrm{g}$

The spatiotemporal fluctuations of small scales in threedimensional turbulent flows are among the most complex phenomena known to classical physics, being both highly non-Gaussian and strongly long-range correlated. For example, velocity gradients as well as velocity increments between two points typically show strong fluctuations much larger than their standard deviation [1,2]. The same is true for fluid accelerations or velocity increments at two different times [2-4], with long-range correlations up to the time scales of the largest eddies in the flow [5]. A possible mechanism for the large fluctuations is the nonlinear self-stretching [6,7] that occurs during the Lagrangian evolution of the velocity gradients, $A_{i j}=$ $\partial_{i} u_{j}$. This local self-stretching must be coupled with the energy exchange among larger or smaller eddies and with velocity fluctuations at different spatial locations via the pressure term. The nonlinear coupling among different scales also relates to the concept of energy cascade, often invoked to explain the growth of non-Gaussianity going from large to small scales.

Significant advances in experimental techniques now allow one to measure all components of $\mathbf{A}$ in different spatial locations [8-10] in high Reynolds number flows. These experimental measurements, together with data generated using direct numerical simulations, have uncovered the existence of many interesting, and possibly universal, geometric features of A. Namely, (i) the preferred alignment of the vorticity vector with the eigenvector of the intermediate eigenvalue of the strain-rate tensor, $\mathbf{S}=(\mathbf{A}+$ $\left.\mathbf{A}^{T}\right) / 2$; (ii) the axisymmetric character of local deformation (two positive and one negative eigenvalue of $\mathbf{S}$ ); (iii) the typical teardrop shape of the joint probability distribution, $P(R, Q)$, where $R=-\operatorname{Tr}\left(\mathbf{A}^{3}\right) / 3$ and $Q=$ $-\operatorname{Tr}\left(\mathbf{A}^{2} / 2\right)$ are two invariants of $\mathbf{A}[7,8,11-13]$.

A systematic analysis of the dynamics of velocity gradients was made by Vieillefosse [6]. He started from the exact equations governing the Lagrangian evolution of $\mathbf{A}$ in the incompressible Navier-Stokes (NS) equations: $\dot{A}_{i j}=$ $-A_{i k} A_{k j}-\partial_{i j} p+\nu \partial^{2} A_{i j}$, where $p$ is the pressure divided by density and $\nu$ the kinematic viscosity. Then, he retained only the isotropic part of the pressure Hessian, $\partial_{i j} p \sim$ $\delta_{i j} \partial^{2} p$, and used the incompressibility condition to express the pressure Laplacian in terms of A. Finally, he neglected the viscous contribution arriving to the closed "restricted Euler" (RE) equations:

$$
\dot{\mathbf{A}}=-\mathbf{A}^{2}+\operatorname{Tr}\left(\mathbf{A}^{2}\right) \mathbf{I} / 3 .
$$

It is remarkable that such a simple system is already sufficient to explain many of the geometrical trends found in the real gradient evolution, as shown by $[6,7]$. On the other hand, the self-stretching mechanism is not constrained by any energy exchange or loss mechanism, leading to a finite-time singularity for almost all initial conditions and preventing the use of the RE dynamics to obtain stationary statistics. Prior models that seek to regularize the RE dynamics include a stochastic model with log-normal statistics of the dissipation [14], a linear and nonlinear damping model for the viscous term [15], and a model where the material deformation history described by a tetrad of points plus some stochastic terms are used to mimic the anisotropic pressure fluctuations [13]. Recently, a model of the anisotropic pressure Hessian and of the viscous term has been proposed by introducing a finitetime memory effect in the closure of the material deformation tensor [16]. Results show that this model reproduces stationarity, non-Gaussianity of transverse and longitudinal gradients, the correct geometrical properties of $\mathbf{A}$, and the experimentally observed relative scaling exponents characterizing how non-Gaussian statistics evolves with Reynolds number, at least from small to moderate values of it. However, attempts to simulate velocity gradient 
dynamics at arbitrarily large Reynolds numbers with this model has been, so far, unsuccessful. It was concluded that the difficulties were associated to the assumptions that the velocity gradient tensor evolves mainly uncoupled from larger or smaller eddies and neighboring locations.

In this Letter we propose a minimal model in which deterministic couplings among scales of motion are included. The model couples the self-stretching local dynamics in the manner of Vieillefosse with a cascade mechanism. The most remarkable result is that such a coupling will be shown to be sufficient to regularize the Vieillefosse finite-time singularity, without destroying the main positive features of (1), including the presence of skewed longitudinal gradients and long tails in the probability density functions of gradients, the vorticity alignment, and the typical teardrop shape of the joint probability, $P(R, Q)$. Also the intermittency level as measured by the flatness coefficient is in good quantitative agreement with experiments $[1,17]$. The model thus provides meaningful results even for very large Reynolds number (here we present results up to $\operatorname{Re}_{\lambda}=1500$ ).

To introduce a cascade dynamics we start from a decomposition of the gradient tensor into bandpassed contributions $\mathbf{A}=\sum_{n} \mathbf{A}_{n}$, each $\mathbf{A}_{n}$ describes the velocity gradient at a typical wave number $k_{n}$ [18]. The set of possible wave numbers is chosen equispaced on a logarithmic scale, $k_{n}=$ $2^{n} k_{0}$. The bandpassed version of the NS equations is of the form

$$
\dot{\mathbf{A}}_{n}=-\sum_{p, q}\left(\mathbf{A}_{p} \mathbf{A}_{q}\right)_{n}+\mathbf{B}_{n}+\nu \partial^{2} \mathbf{A}_{n}
$$

where a further projection on the traceless part of the righthand side must be assumed and the term $\mathbf{B}_{n}$ represents pressure effects, interscale interactions, and additional spatial transport introduced by the bandpass filtering acting on the advective term on the left-hand side of the original equation. Neglecting the viscous term and $\mathbf{B}_{n}$, and keeping only the fully diagonal term $(p=q=n)$ in the double sum, leads to the most severe approximation. Namely, that the gradients $\mathbf{A}_{n}$, bandpassed on different shells, follow the RE dynamics separately shell-by-shell: $\dot{\mathbf{A}}_{n}=-\mathbf{A}_{n}^{2}+$ $\operatorname{Tr}\left(\mathbf{A}_{n}^{2}\right) \mathbf{I} / 3$. Of course this set of uncoupled equations suffers from the same drawbacks of the original Vieillefosse model. The coupling between different scales must be introduced by keeping some additional terms besides the purely diagonal one; i.e., we include a new term $\mathbf{F}$ and we choose its form by imposing an energy preserving structure, following the assumption that the pressureHessian does not perform work on the bandpassed gradient [13]. Moreover, it is natural to suppose that the most relevant dynamical interactions happen within nearby scales and therefore we limit the range of interactions to the next-nearest neighbors. One possible choice for the coupling term on the $n$th shell is

$$
\begin{aligned}
\mathbf{F}_{n}= & \mathbf{A}_{n+2} \mathbf{A}_{n+1}^{T}+b 2^{2} \mathbf{A}_{n-1}^{T} \mathbf{A}_{n+1} \\
& +(1-b) 2^{4} \mathbf{A}_{n-2} \mathbf{A}_{n-1},
\end{aligned}
$$

where $b$ is a free parameter (always fixed to $b=0.5$ hereafter). Let us notice that the structure of $\mathbf{F}_{n}$ is chosen such that the total energy $E=\sum_{n} k_{n}^{-2} \operatorname{Tr}\left(\mathbf{A}_{n} \mathbf{A}_{n}^{T}\right)$ is preserved by the effects of the three nonlinear terms. This specific form is proposed as a model for the neglect of all the truncated terms in the double sum and the $\mathbf{B}_{n}$ transport term. The functional form proposed for $\mathbf{F}_{n}$ is motivated by the typical structure of the nonlinear terms used in Shell models of turbulence [19]. The viscous Laplacian term in NS equations is modeled using a linear damping term on each band proportional to $\nu k_{n}^{2}$, where $\nu$ is the viscosity. Combining all these elements one obtains the following dynamical system:

$$
\dot{\mathbf{A}}_{n}=\alpha\left[-\mathbf{A}_{n}^{2}+1 / 3 \operatorname{Tr}\left(\mathbf{A}_{n}^{2}\right) \mathbf{I}\right]+(1-\alpha)\left[\tilde{\mathbf{F}}_{n}-\nu k_{n}^{2} \mathbf{A}_{n}\right],
$$

where $\tilde{\mathbf{F}}_{n} \equiv \mathbf{F}_{n}-1 / 3 \operatorname{Tr}\left(\mathbf{F}_{n}\right) \mathbf{I}$ is the traceless part of $\mathbf{F}_{n}$ and where $\alpha$ is a parameter which weights the relative importance of the RE dynamics with respect to the energy exchange and energy dissipation terms. As a first step, in this work we restrict attention to the case $\alpha=0.5$. In the previous equations the infrared and ultraviolet truncation is imposed by keeping in the nonlinear term $\mathbf{A}_{n}=0$ for $n=$ $\{-1,-2, N+1, N+2\}$. Note that the present model for velocity gradient does not make predictions about the spatial distribution $\mathbf{A}_{n}(\mathbf{x})$ or of its spatial gradients $\left(\nabla \mathbf{A}_{n}\right.$, i.e., higher-order derivatives of the velocity).

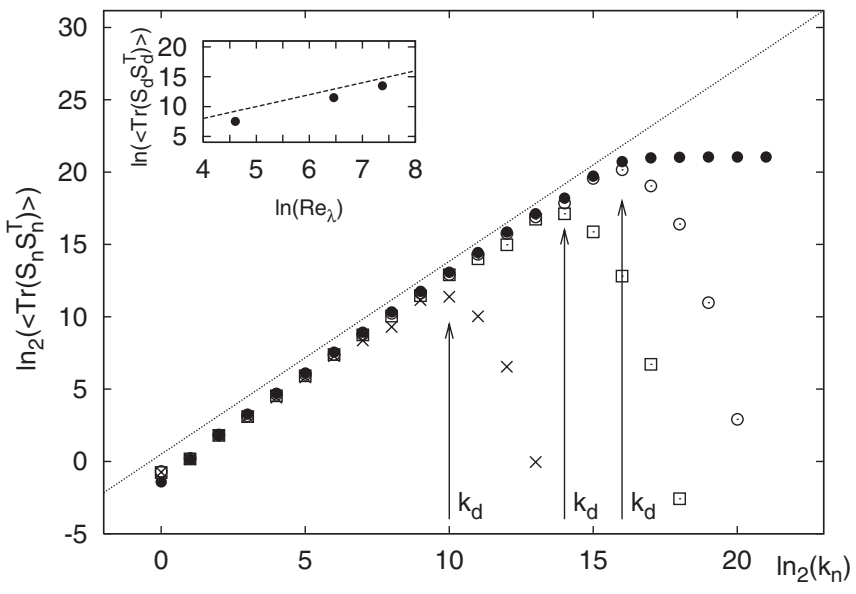

FIG. 1. Log-log plot of the bandpassed energy dissipation $\mathcal{E}_{n}$ vs $k_{n}$, for $\operatorname{Re}_{\lambda}=1500(\bigcirc) ; \operatorname{Re}_{\lambda}=640(\square) ; \operatorname{Re}_{\lambda}=130(\times)$. The dissipative scale $k_{d}$ is defined as the wave number where the energy dissipation peaks (arrows in the plot). The straight line corresponds to the Kolmogorov spectrum. The data which saturates in the viscous range $(\mathbf{O})$ are for the coarse-grained variables (defined later): $\left\langle\operatorname{Tr}\left(\mathbf{S}_{n}^{\mathrm{CG}} \mathbf{S}_{n}^{\mathrm{CG}}\right)\right\rangle$. In the inset we show the Reynolds dependency of $\mathcal{E}_{n}$ at $k_{n}=k_{d}$. The straight line corresponds to the expected slope $\propto \operatorname{Re}_{\lambda}^{2}$. 
Thus, differential constraints such as $\nabla \times \mathbf{A}_{n}=0$ cannot be directly enforced in the model.

The main question is if a stationary statistical state can be achieved once a forcing is applied at large scales, i.e., if the simple energy-exchange term $\mathbf{F}_{n}$ is sufficient to regularize the dynamics of the RE structure at all scales. This will allow us also to study the important questions related to the dependency of the geometrical structure of turbulence on the Reynolds numbers and its correlation with inertial range quantities. The simplest way to achieve a stationary state is to add a statistically homogeneous and isotropic white-in-time Gaussian tensor $\mathbf{G}$ at the largest band $n=0$, with covariance $\left\langle G_{i j} G_{m l}\right\rangle=2 \delta_{i m} \delta_{j l}-$ $1 / 2 \delta_{i j} \delta_{m l}-1 / 2 \delta_{i l} \delta_{j m}[16]$.

The gradient statistics will be studied by looking at the shell $k_{n}=k_{d}$, defined as where the spectrum of the $\mathbf{A}_{n}$ has its peak, i.e., where a balance between the quadratic terms and the viscous terms of (4) is obtained. The system of equations is integrated numerically using a 4th order Runge-Kutta scheme. Three Reynolds numbers are simulated by using $\nu=\left\{10^{-4}, 2.7 \times 10^{-6}, 4.4 \times 10^{-7}\right\}$ and with a total number of shells $N=\{14,18,22\}$, corresponding to $\operatorname{Re}_{\lambda}=\{130,640,1500\}$, respectively. Results are shown in Fig. 1, which shows the time-averaged energy dissipation (without multiplication by $\nu$ ), measured on the bandpass gradient variables, $\mathcal{E}_{n}=\left\langle\operatorname{Tr}\left(\mathbf{A}_{n} \mathbf{A}_{n}^{T}\right)\right\rangle$ as a function of $k_{n}$ : notice that it has the expected Kolmogorov scaling $\left\langle\operatorname{Tr}\left(\mathbf{A}_{n} \mathbf{A}_{n}^{T}\right)\right\rangle \sim\left\langle\operatorname{Tr}\left(\mathbf{S}_{n} \mathbf{S}_{n}^{T}\right)\right\rangle \sim k_{n}^{4 / 3}$.

On dimensional grounds we expect that, at the wave number where the matching is achieved, $\left\langle\operatorname{Tr}\left(S_{d} S_{d}^{T}\right)\right\rangle$ scales with Reynolds number as $\operatorname{Re}_{\lambda}^{2}$. This is indeed verified in the inset of Fig. 1. Geometrical properties of small-scale turbulent fluctuations can be monitored by studying the joint statistics of the two invariants $Q, R$ at different Reynolds number and/or at different scales. Here, by measuring the simultaneous distribution of $Q_{n}=-\operatorname{Tr}\left(\mathbf{A}_{n}^{2}\right) / 2$ and of $R_{n}=-\operatorname{Tr}\left(\mathbf{A}_{n}^{3}\right) / 3$ for different Reynolds numbers, we can do both. In Fig. 2 we show the isolines of the joint probability distribution $P\left(R_{n}, Q_{n}\right)$ measured on the dissi-
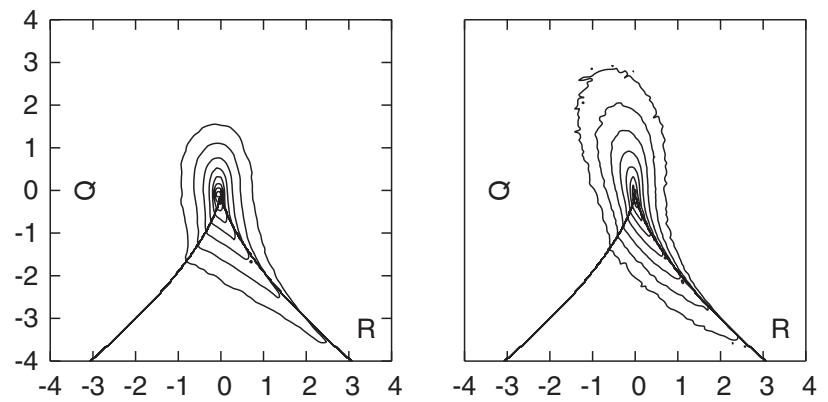

FIG. 2. Isolines of $P(R, Q)$ (normalized to have unit variance) for DNS and model data. Right-hand panel: DNS data at $\operatorname{Re}_{\lambda} \sim$ 180. Left-hand panel: model data at $\operatorname{Re}_{\lambda}=130$, evaluated at the dissipative scale $k_{d}(\mathrm{Re})$. The continuous line in all diagrams corresponds to the Vieillefosse zero-discriminant curve $Q^{3}+$ $27 / 4 R^{2}=0$. pative scale $n=d$. We notice the presence of high probable fluctuations along the right Vieillefosse tail, also seen in real turbulence, which is shown in the right-hand panel of Fig. 2. The data are from direct numerical simulation (DNS), done at a similar Reynolds number. As can be seen, there is very good agreement of the model with the NavierStokes data, except for a small depletion of events in the third quadrant $(R<0, Q>0)$. In this quadrant, real data are strongly affected by vortex stretching, an effect which is evidently underrepresented by the model evolution. The $P(R, Q)$ distribution has very little sensitivity to Reynolds number (at least in the range investigated here). The $P(R, Q)$ distribution becomes more symmetric for bandpass variables at larger scales (not shown), and this effect is also observed in Navier-Stokes turbulence [12,13]. Finally, in Fig. 3 we present quantification of the alignment between the vorticity at the dissipative scale, $\left(w_{i}\right)_{d}=$ $\epsilon_{i j l}\left(A_{j l}\right)_{d}$, and the three eigenvectors of the strain-rate tensor $\mathbf{S}_{d}$ also at that scale. It is well known from DNS and experiments that vorticity tends to preferentially align with the intermediate eigenvector [8,9,20]: Fig. 3 shows that our model is able to also capture this feature. Finally, we document the results in terms of intermittency. First, we show in the inset of Fig. 4 the probability distribution function (PDF) of both longitudinal and transverse gradients. Correctly, the model possesses longitudinal skewed distribution while the transverse gradient PDF is fully symmetric. The gradient fluctuations are highly nonGaussian as a result of the growth of intermittency going from the large scale down to the dissipative scale. This growth at decreasing length scales is often characterized by measuring the flatness coefficient of velocity gradients, $\mathcal{F}_{4}=\left\langle A_{d}^{4}\right\rangle /\left\langle A_{d}^{2}\right\rangle^{2}$, and plotting it as a function of Reynolds number $\operatorname{Re}_{\lambda}$, where with $A_{d}$ we mean any of the longitudinal components of $\mathbf{A}$.

As a technical difficulty we point out that the exponential falloff of the gradient spectrum in the shell-matrix model (4) prevents us from a direct measurement of $\mathcal{F}_{4}$ on the dissipative shell (the flatness tends to grow exponentially in the dissipative range, making the estimate very delicate). For that reason we decide to measure the flatness using a coarse-grained variable. Specifically, the coarse-
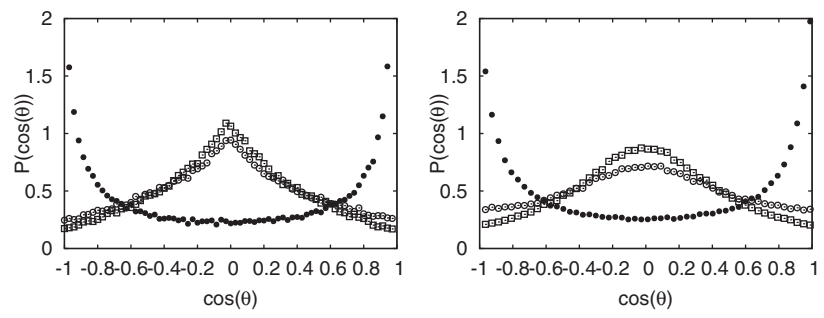

FIG. 3. PDF of the cosine between the vorticity and the eigenvectors associated with the intermediate $(\bigcirc)$, maximum $(\bigcirc)$, and smallest $(\square)$ eigenvalues of the strain-rate matrix. Notice the good agreement between the DNS (right) data and the model data (left). 


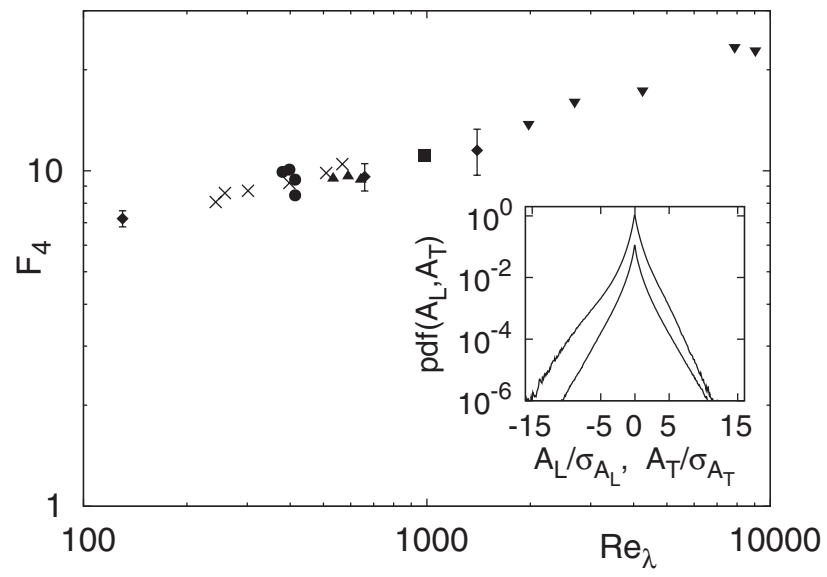

FIG. 4. Comparison between a collection of experimental data for the longitudinal gradient flatness [17], superposed with the results from our model (diamonds with error bars). We have used the variance of the longitudinal coarse-grained gradient, $\sigma_{A}=$ $\left\langle\left(A_{11}^{\mathrm{CG}}\right)^{2}\right\rangle^{1 / 2}$, to define the Reynolds number: $\operatorname{Re}_{\lambda}=2 E /\left(3 \nu \sigma_{A}\right)$ [2]. Inset: Data for $\operatorname{Re}_{\lambda}=1500$. Normalized PDF of longitudinal (top) and transverse (bottom) bandpassed gradients measured at $k_{d}$. Notice the skewed profile for the longitudinal gradients. Longitudinal PDF has been shifted along the $y$ axis for sake of clarity.

grained velocity gradient at scale $k_{m}$ is defined as $\mathbf{A}_{m}^{\mathrm{CG}}=$ $\sum_{n=0}^{n=m} \mathbf{A}_{n}$. For large $m$, we obtain a variable which includes fluctuations on all scales, and which becomes independent on $m$ (saturates) beyond the viscous range. The two variables $\mathbf{A}_{n}$ and $\mathbf{A}_{n}^{\mathrm{CG}}$ have obviously the same scaling in the inertial range. The bandpassed variable decays exponentially in the dissipative range while the coarse-grained one saturates; see Fig. 1. This saturation allows us to have robust measurements of intermittency in the dissipative range. In the main body of Fig. 4 we show the behavior of flatness of the coarse-grained velocity gradient as a function of Reynolds number, superposed with experimental data. Within the range of Reynolds numbers considered, the agreement is very satisfactory.

In conclusion, we have introduced a "shell" version of the RE dynamics which is free from the finite-time singularity of the original Vieillefosse formulation. The regularization is achieved at all scales thanks to the introduction of an energy-exchange mechanism between shells. The model shows very realistic behavior at changing Reynolds numbers, including (i) the skewed nature of longitudinal gradients, (ii) the alignment of vorticity with the intermediate eigenvector of the strain-rate tensor, (iii) the accumulation of events along the right tail of the Vieillefosse line, and (iv) the correct level of intermittency as measured by the flatness and its increasing trend at increasing $\mathrm{Re}_{\lambda}$. A set of questions remains open. From a dynamical point of view, the model seems to underpredict the probability of observing strong vortex stretching events - this could point to open challenges associated with modeling small-scale coherent vortices [4]. A prom- ising development could be to couple the present multiscale approach with the Lagrangian deformation method proposed in [16]. The multiscale model presented here could possibly be generalized and applied to study additional effects in turbulence. Examples could include scalar gradient vector, helical properties (models for the joint evolution of velocity and vorticity), buoyancy effects (including a scalar-dependent forcing term), magnetohydrodynamic effects (model for the gradient of Elsasser variables), and multiphase flow (e.g., relating shear statistics to the breakup of droplets or bubbles as function of scale).

We thank R. Benzi for helpful discussions. L. B. and F. T. acknowledge the hospitality of the Center for Environmental and Applied Fluid Mechanics of Johns Hopkins University where part of this work was done. L. C. acknowledges the support of the Keck Foundation, C.M. thanks the support of the U.S. National Science Foundation, F. T. thanks CNR for support under the Short Term Mobility grant.

[1] K. R. Sreenivasan and R. Antonia, Annu. Rev. Fluid Mech. 29, 435 (1997).

[2] U. Frisch, Turbulence: The Legacy of A. N. Kolmogorov (Cambridge University, Cambridge, England, 1995).

[3] A. La Porta et al., Nature (London) 409, 1017 (2001); J. Fluid Mech. 469, 121 (2002).

[4] L. Biferale et al., Phys. Fluids 17, 021701 (2005); L. Chevillard et al., Phys. Rev. Lett. 91, 214502 (2003).

[5] N. Mordant, E. Leveque, and J.-F. Pinton, New J. Phys. 6, 116 (2004).

[6] P. Vieillefosse, Physica (Amsterdam) 125A, 150 (1984).

[7] B. J. Cantwell, Phys. Fluids A 5, 2008 (1993); Phys. Fluids A 4, 782 (1992).

[8] A. Tsinober, E. Kit, and T. Dracos, J. Fluid Mech. 242, 169 (1992);

B. Luthi, A. Tsinober, and W. Kinzelbach, J. Fluid Mech. 528, 87 (2005).

[9] B. Tao, J. Katz, and C. Meneveau, J. Fluid Mech. 457, 35 (2002).

[10] B. W. Zeff et al., Nature (London) 421, 146 (2003).

[11] T. S. Lund and M. M. Rogers, Phys. Fluids 6, 1838 (1994).

[12] F. van der Bos et al., Phys. Fluids 14, 2456 (2002).

[13] M. Chertkov, A. Pumir, and B. Shraiman, Phys. Fluids 11, 2394 (1999); A. Naso and A. Pumir, Phys. Rev. E 72, 056318 (2005).

[14] S. S. Girimaji and S. B. Pope, Phys. Fluids A 2, 242 (1990).

[15] J. Martin et al., Phys. Fluids 10, 2336 (1998); E. Jeong and S. S. Girimaji, Theor. Comput. Fluid Dyn. 16, 421 (2003).

[16] L. Chevillard and C. Meneveau, Phys. Rev. Lett. 97, 174501 (2006).

[17] R. A. Antonia, A. J. Chambers, and B. R. Satyaprakash Bound.-Lay. Meteorol. 21, 159 (1981).

[18] G. Eyink, J. Fluid Mech. 549, 159 (2006).

[19] L. Biferale, Annu. Rev. Fluid Mech. 35, 441 (2003).

[20] W. T. Ashurst et al., Phys. Fluids 30, 2343 (1987). 